\title{
ON SUBVARIETIES OF A HERMITIAN MANIFOLD
}

\author{
CARLOS J. FERRARIS
}

\begin{abstract}
We make use of the variational properties of the geodesic distance function of a Riemannian manifold and the technique of the "blowing-up" ( $\sigma$-process) on a complex manifold to derive the nonexistence of compact complex analytic subvarieties in a simply connected, complete Hermitian manifold with nonpositive sectional curvature.
\end{abstract}

1. Notation and preliminaries. In this section we introduce the notation and definitions that will be used in the following sections.

(i) Let $M^{n}$ be a complete connected Riemannian manifold of dimension $n$ and class $C^{\infty}$. Throughout this note we will indicate by

$r=$ the geodesic distance function of $M$.

$M_{\Delta}=$ the diagonal of the product manifold $M \times M$.

$M_{\text {cut }}=$ the set of all cut pairs of $M$.

$M \vee M=M \times M \backslash M_{\Delta} \cup M_{\text {cut }}$.

$W_{c}=\{(p, q) \in M \times M / r(p, q)=c\}$, for every positive real number $c$.

$\mathrm{II}_{W_{c}}=$ the second fundamental form of $W_{c}$ (we observe that $W_{c} \cap M \vee$ $M$ is a submanifold of $M \vee M$ of codimension 1) with grad $r$ taken as the normal vector to $W_{c}$.

For every $(p, q)$ in $M \vee M$ we introduce a natural orthonormal frame of $M \vee M$ at $(p, q)$ as follows:

Let $\gamma$ be the unique geodesic (parametrized by arc-length) in $M$ between $p$ and $q$ and let $\left\{e_{i}^{\prime}(p), 1 \leqslant i \leqslant n\right\}$ be an orthonormal frame of $M$ at $p$, with $e_{1}^{\prime}(p)=$ the tangent vector to $\gamma$ at $p$; by parallel translation along $\gamma$ from $p$ to $q$ we construct an orthonormal frame $\left\{e_{i}^{\prime \prime}(q), 1<i<n\right\}$ of $M$ at $q$ and then we set

$$
e_{ \pm i}(p, q)=\frac{1}{\sqrt{2}}\left[\left(e_{i}^{\prime}(p), 0\right) \pm\left(0, e_{i}^{\prime \prime}(q)\right)\right], \quad 1<i<n .
$$

We introduce the subspaces

$$
V_{(p, q)}^{+}=\sum_{i=1}^{n} \mathbf{R} e_{i}(p, q) \quad \text { and } \quad V_{(p, q)}^{-}=\sum_{i=1}^{n} \mathbf{R} e_{-i}(p, q)
$$

Received by the editors May 23, 1978.

AMS (MOS) subject classifications (1970). Primary 53C55; Secondary 32C25.

Key words and phrases. Riemannian manifold, Hermitian manifold, sectional curvature, complex analytic subvariety, "blowing-up", projective tangent cone.

(C) 1979 American Mathematical Society 0002-9939/79/0000-0069/\$02.75 
and observe that for $(p, q)$ in $M \vee M$

$$
T_{(p, q)} W_{c}=V_{(p, q)}^{+} \oplus V_{(p, q)}^{-} \backslash \mathbf{R} e_{-1}(p, q),
$$

where $c=r(p, q)$. Moreover, $e_{1}(p, q)$ is a principal direction of curvature of $W_{c}$ at $(p, q)$ with normal curvature equal to zero, see [2].

(ii) Let $M$ be a complex manifold and let $p$ be a point in $M$. We will denote by $\hat{M}_{p}$ the "blowing-up" manifold of $M$ at $p$, by $\sigma: \hat{M}_{p} \rightarrow M$ the corresponding quadratic transformation and by $B_{p}=\sigma^{-1}(\{p\})$. Notice that $B_{p}$ is isomorphic to a complex projective space $\mathbf{C} P^{n-1}, n=\operatorname{dim}_{c} M$. Let $V$ be a complex analytic subvariety of $M$ with $p$ in $V$, then the set $\hat{V}_{p}$ (topological closure of $\sigma^{-1}(V \backslash\{p\})$ in $\left.\hat{M}_{p}\right)$ is a subvariety of $\hat{M}_{p}$ and it is called the "blowing-up" of the variety $V$ at $p$.

The projective tangent cone of $V$ at $p$ is defined by

$$
K_{p}(V)=\hat{V}_{p} \cap B_{p} \text {. }
$$

It is known that if $V$ is an irreducible variety of dimension $d$ then $K_{p}(V)$ is a variety of dimension $d-1$.

2. Manifolds with nonpositive sectional curvature. In this section we will make use of the variational properties of the geodesic distance function $r$ to derive certain properties about the "position" of the submanifolds $W_{c}$ when $M$ has nonpositive sectional curvature. We start by proving

Proposition 1. Let $M^{n}$ be a simply connected, complete Riemannian manifold with nonpositive sectional curvature. Then for every real number $c>0$, the submanifold $W_{c}$ has at every point $2 n-2$ principal directions in which the normal curvature is negative and one principal direction with curvature zero.

Proof. Let $(p, q) \in M \vee M$ and set $c=r(p, q)$. Let $\gamma$ be the unique geodesic (parametrized by arc-length) in $M$ between $p$ and $q$. For every $v \in\left(\operatorname{Re}_{1}(p, q)\right)^{\perp}$ (the orthogonal complement to $\operatorname{Re}_{1}(p, q)$ in $\left.T_{(p, q)} W_{c}\right)$ we construct a variation $W(s, t), 0 \leqslant s \leqslant c,-\infty<t<\infty$, satisfying:

(a) $W(s, 0)=\gamma(s), 0 \leqslant s \leqslant c$,

(b) $W(0, t)=\exp _{p}\left(t v_{1}\right)=p_{t}$ and $W(c, t)=\exp _{q}\left(t v_{2}\right)=q_{t}$, where $v_{1} \in$ $T_{p} M \backslash \mathbf{R} e_{1}^{\prime}(p), v_{2} \in T_{q} M \backslash \mathbf{R} e_{1}^{\prime \prime}(q)$ and $v=v_{1}+v_{2}$.

(c) For each $t, W(t, \cdot)$ is the unique geodesic (parametrized by arc-length) in $M$ between $p_{t}$ and $q_{t}$.

From the fact that $M$ is simply connected and has nonpositive curvature it follows that such variation exists and can be represented as

$$
W(s, t)=\exp _{p_{t}}\left(\frac{\operatorname{sr}\left(p_{t}, q_{t}\right)}{c} \tilde{v}\left(p_{t}, q_{t}\right)\right)
$$

where $\tilde{v}\left(p_{t}, q_{t}\right) \in T_{p_{t}}(M),\left\|\tilde{v}\left(p_{t}, q_{t}\right)\right\|=1$. Observe that $\tilde{v}\left(p_{t}, q_{t}\right)$ depends differentiably on $p_{t}$ and $q_{t}$. Therefore $W(s, t)$ is a differentiable variation of $\gamma$ by geodesics with

$$
L(W(\cdot, t))=r\left(p_{t}, q_{t}\right)=r\left(\exp _{(p, q)} t v\right) \text { for all } t,
$$


and

$$
\left.\frac{d}{d t} L(W(\cdot, t))\right|_{t=0}=0
$$

On the other hand the formula for the second variation is [1]

$$
\left.\frac{d^{2}}{d t^{2}} L(W(\cdot, t))\right|_{t=0}=\left.\int_{0}^{c}\left(\left\|Y^{\prime}\right\|^{2}-K(T, Y)\|Y\|^{2}-\left\langle T, Y^{\prime}\right\rangle^{2}\right)\right|_{t=0} d s,
$$

where $Y=\partial W / \partial t, T=\partial W / \partial s, Y^{\prime}=D Y / d s$ (covariant derivative), and $K(T, Y)$ stands for the sectional curvature of the plane spanned by $T$ and $Y$. We claim that in our case $\left.\left\langle T, Y^{\prime}\right\rangle\right|_{t=0}=0$. In fact

$$
\begin{aligned}
\left.\left\langle T, Y^{\prime}\right\rangle\right|_{t=0} & =\left.\left\langle\frac{\partial W}{\partial s}, \frac{D}{\partial s} \frac{\partial W}{\partial t}\right\rangle\right|_{t=0} \\
& =\left.\left\langle\frac{\partial W}{\partial s}, \frac{D}{\partial t} \frac{\partial W}{\partial s}\right\rangle\right|_{t=0}=\left.\frac{1}{2} \frac{\partial}{\partial t}\left\langle\frac{\partial W}{\partial s}, \frac{\partial W}{\partial s}\right\rangle\right|_{t=0} \\
& =\left.\frac{1}{2} \frac{d}{d t}\left\langle r\left(p_{t}, q_{t}\right) \tilde{v}, r\left(p_{t}, q_{t}\right) \tilde{v}\right\rangle\right|_{t=0}=\left.\frac{1}{2} \frac{d}{d t} r^{2}\left(p_{t}, q_{t}\right)\right|_{t=0}
\end{aligned}
$$

which is zero because of (2). Hence the second variation formula becomes

$$
\left.\frac{d^{2}}{d t^{2}} L(W(\cdot, t))\right|_{t=0}=\left.\int_{0}^{c}\left(\left\|Y^{\prime}\right\|^{2}-K(T, Y)\|Y\|^{2}\right)\right|_{t=0} d s .
$$

Applying (3) to the $2 n-2$ independent directions $\left\{e_{i}(p, q)-e_{-i}(p, q)\right.$; $\left.e_{-i}(p, q), 2 \leqslant i \leqslant n\right\}$ we see that for each of them the second variation formula is strictly positive. This, together with the fact that because of (1),

$$
\left.\frac{d^{2}}{d t^{2}} L(W(\cdot, t))\right|_{t=0}=\left.\frac{d^{2}}{d t^{2}} r\left(p_{t}, q_{t}\right)\right|_{t=0}
$$

and

$$
\left.\frac{d^{2}}{d t^{2}} r\left(p_{t}, q_{t}\right)\right|_{t=0}=-\mathrm{II}_{W_{c}}[v]
$$

tells us that $W_{c}$ has $2 n-2$ principal directions with negative curvature and one principal direction with zero curvature (namely, $e_{1}$ ). This proves the proposition. Now we are able to prove

TheORem 1. Let $M^{n}$ be as in Proposition 1 and let $(p, q)$ in $M \vee M$, with $c=r(p, q)$. Then there exists $\varepsilon>0$ sufficiently small, such that

$$
r(p, q) \leqslant r\left(\exp _{(p, q)} v\right)
$$

for all $v$ in $B_{\varepsilon}(0)$, where $B_{\varepsilon}(0)$ is the ball with center at 0 and radius $\varepsilon$ in $T_{(p, q)} W_{c}$.

Proof. Let $f_{1}, f_{2}, \ldots, f_{2 n-1}$ with $f_{1}=e_{1}$ be the orthonormal frame of $W_{c}$ at $(p, q)$ formed by the principal directions with normal curvatures $k_{1}=0$ and $k_{i}\left\langle 0, i \geqslant 2\right.$. Let us take $\delta>0$ so small that $\exp _{(p, q)}$ restricted to the ball 
$B_{\delta}(0)$ in $T_{(p, q)} M \times M$ is a diffeomorphism and then set

$$
S_{\delta}=\exp _{(p, q)}\left(B_{\delta}(0) \cap T_{(p, q)} W_{c}\right) .
$$

We consider on $S_{\delta}$ coordinates $u_{i}$ defined by

$$
u_{i}\left[\exp _{(p, q)}\left(\sum_{j=1}^{2 n-1} \lambda_{j} f_{j}\right)\right]=\lambda_{i}, \quad 1 \leqslant i \leqslant 2 n-1 .
$$

Denote by $\left(p_{s}, q_{s}\right)$ a point in $S_{\delta}$ satisfying:

$$
u_{1}\left(p_{s}, q_{s}\right)=s, \quad u_{i}\left(p_{s}, q_{s}\right)=0, \quad i \geqslant 2 \text {. }
$$

We first prove that

$$
d r_{\left(p_{s}, q_{s}\right)} \mid T_{\left(p_{s}, q_{s}\right)} S_{\delta}=0 .
$$

In fact, for every curve $\alpha(t),|t|<\rho$, in $S_{\delta}$ that goes through the point $\left(p_{s}, q_{s}\right)$, i.e. $\alpha(0)=\left(p_{s}, q_{s}\right)$, we have

$$
\alpha(t)=\exp _{(p, q)} v(t), \quad|t|<\rho,
$$

where $v(t) \in T_{(p, q)} W_{c}$.

On the other hand

$$
T_{(p, q)} W_{c}=V_{(p, q)}^{+} \oplus V_{(p, q)}^{-} \backslash \mathbf{R} e_{-1}(p, q),
$$

hence

$$
v(t)=\lambda_{1}(t) e_{1}(p, q)+\sum_{i=2}^{n} \lambda_{i}(t) e_{i}(p, q)+\sum_{j=2}^{n} \mu_{j}(t) e_{-j}(p, q)
$$

Thus

$$
\begin{aligned}
\alpha(t)= & \exp _{p}\left(\lambda_{1}(t) e_{1}^{\prime}(p)+\text { something perpendicular to } e_{1}^{\prime}(p)\right) \\
& \times \exp _{q}\left(\lambda_{1}(t) e_{1}^{\prime \prime}(q)+\text { something perpendicular to } e_{1}^{\prime \prime}(q)\right),
\end{aligned}
$$

but then

$$
\left.\dot{\alpha}(0)=\dot{\lambda}_{1}(0) e_{1}\left(p_{s}, q_{s}\right)+\text { something in } V_{\left(p_{s}, q_{s}\right)}^{+}\right) \backslash \mathbf{R} e_{1} \oplus V_{\left(p_{s}, q_{s}\right)}^{-} \backslash \mathbf{R} e_{-1}
$$

which means that

$$
T_{\left(p_{s}, q_{s}\right)} S_{\delta} \subseteq T_{\left(p_{s}, q_{s}\right)} W_{r\left(p_{s}, q_{s}\right)} .
$$

By dimensional reasons equality must hold and this proves (4). It follows from (4) that the coordinates $u_{1}, \ldots, u_{2 n-1}$ can be written as

$$
r(\bar{p}, \bar{q})=r(p, q)+\frac{1}{2} \sum_{i=2}^{2 n-1} k_{i} u_{i}^{2}+\sum_{i_{1} j=2}^{2 n-1} u_{i} u_{j} \varphi_{i j}(u)
$$

where each $\varphi_{i j}(0)=0$. Hence there exists $\varepsilon>0$ sufficiently small such that

$$
r(\bar{p}, \bar{q}) \geqslant r(p, q) \text { for all }(\bar{p}, \bar{q}) \text { in } S_{\varepsilon} .
$$

This completes the proof of Theorem 1 .

Next we apply Theorem 1 to obtain 
Theorem 2. Let $M^{n}$ be as in Proposition 1. Then the geodesic distance function $r$ of $M^{n}$ cannot achieve a positive relative maximum on any $k$-dimensional $(k>1)$ totally geodesic submanifold $V^{k}$ in $M \times M$.

Proof. Let $V^{k}(k>1)$ be a totally geodesic submanifold in $M \times M$ and let us assume that $r$ achieves a positive relative maximum $c$ on $V^{k}$ at $(p, q)$. We consider through $(p, q)$ the $2 n-1$ dimensional submanifold $S_{e}$ given by Theorem 1. Then from the definition of $S_{\varepsilon}$ we have

$$
T_{(p, q)} S_{\varepsilon}=T_{(p, q)} W_{c}
$$

and on the other hand, because of the maximality of $r$ on $V^{k}$ at $(p, q)$,

$$
T_{(p, q)} V^{k} \subseteq T_{(p, q)} W_{c}
$$

From (5) and (6) and the fact that $k>1$, there exists $0 \neq v \in T_{(p, q)} V^{k} \backslash \mathbf{R} e_{1}$ and then by Theorem 1

$$
r(p, q)<r\left(\exp _{(p, q)} t v\right) \text { for } t \text { small. }
$$

But since $V^{k}$ is totally geodesic, $\exp _{(p, q)} t v \in V^{k}$, for all $t$, and because of the local maximality of $r$ on $V^{k}$ at $(p, q)$,

$$
r(p, q) \geqslant r\left(\exp _{(p, q)} t v\right)
$$

which contradicts (7). Thus $r$ cannot achieve a positive relative maximum on $V^{k}$ which was our assertion.

We observe that from Theorem 2 follows the well-known fact that there exists no compact totally geodesic submanifold on a simply connected, complete Riemannian manifold with nonpositive curvature.

3. Hermitian manifolds with nonpositive sectional curvature. In this section we make use of Proposition 1 and the technique developed in [2], to obtain our main result, namely

THEOREM 3. Let $M$ be a hermitian manifold of complex dimension $n$, simply connected, complete and with nonpositive sectional curvature. Then $M \vee M$ has no compact complex analytic subvarieties of complex dimension $k>1$.

Proof. Let us assume that there exists $V^{k}$ a compact complex analytic subvariety of $M \vee M(k>1)$. Then $r$ has a maximum on $V^{k}$ at $(p, q)$, $r(p, q)=c$. We know that the tangent space of $W_{c}$ at $(p, q)$ can be written as

$$
T_{(p, q)} W_{c}=\sum_{i=1}^{n} \mathbf{R} e_{i} \oplus \sum_{i=1}^{n} \mathbf{R} J e_{i} \oplus \sum_{i=2}^{n} \mathbf{R} e_{-i} \oplus \sum_{i=1}^{n} \mathbf{R} J e_{-i}
$$

Now, we consider the following complex subspace of $T_{(p, q)} W_{c}$,

$$
T_{(p, q)} W_{c} \cap J\left(T_{(p, q)} W_{c}\right)
$$

and take a complex submanifold $W$ through $(p, q)$ such that

$$
T_{(p, q)} W=T_{(p, q)} W_{c} \cap J\left(T_{(p, q)} W_{c}\right) .
$$

Let $K_{(p, q)} W$ and $K_{(p, q)} V^{k}$ be the projective tangent cones of $W$ and $V^{k}$, 
respectively. Since $r$ has a maximum on $V^{k}$ at $(p, q)$,

$$
K_{(p, q)} V^{k} \subseteq K_{(p, q)} W .
$$

We may assume that $V^{k}$ is an irreducible subvariety and then $K_{(p, q)} V^{k}$ has dimension $k-1>0$. Therefore there exists $A_{0}$ in $K_{(p, q)} V^{k}$ which corresponds to a direction of $T_{(p, q)} W_{c} \cap J\left(T_{(p, q)} W_{c}\right)$. On the other hand since $V_{(p, q)}^{k}$

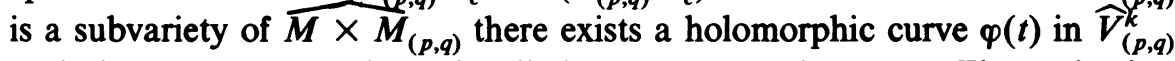
such that $\varphi(0)=A_{0}$ and $\varphi(t)$ locally intersects $B_{(p, q)}$ just at $A_{0}$. The projection of $\varphi(t)$ under the quadratic transformation $\sigma$ to $M \times M$ gives us a holomorphic curve $C(t)$ in $V^{k}$ with $C(0)=(p, q)$, and such that if $\left(z^{1}, \ldots, z^{2 n}\right)$ is a coordinate system in a neighborhood of $(p, q)$ with $z^{i}(p, q)$ $=0,1<i<2 n$, there exists an integer $d \geqslant 1$ such that

$$
z^{\alpha}(C(t))=z^{\alpha}(t)=\sum_{j=0}^{\infty} A_{j}^{\alpha} t^{d+j}, \quad 1 \leqslant \alpha \leqslant 2 n ;
$$

here the $2 n$-tuple $\left(A_{0}^{1}, \ldots, A_{0}^{2 n}\right)$ corresponds to the local homogeneous coordinates of $A_{0}$ in $\overline{M \times M_{(p, q)}}$.

We are going to show that for every $t \in \mathbf{C}$, with $|t|$ small, there exists $\zeta_{t}$ with $\left|\xi_{t}\right|=|t|$ and

$$
r\left(C\left(\zeta_{t}\right)\right)-r(C(0))>0 .
$$

In fact, let us take the average of the function $r(C(t))-r(C(0))$. From Theorem 3 of [2], we know that

$$
\frac{1}{2 \mathrm{II}} \int_{0}^{2 \mathrm{III}}\left(r\left(C\left(t e^{i \theta}\right)\right)-r(C(0))\right) d \theta=r_{, \alpha \bar{\beta}}\left(\sum_{j=0}^{\infty} A_{j}^{\alpha} \overline{A_{j}^{\beta}}|t|^{2(d+j)}\right)
$$

where $r_{, \alpha \bar{\beta}}$ stands for component of the second covariant derivative of $r$. On the other hand.

$$
-2 r_{, \alpha \bar{\beta}} A_{0}^{\alpha} \overline{A_{0}^{\beta}}=\mathrm{II}_{W_{c}}\left[A_{0}\right]+\mathrm{II}_{W_{c}}\left[J A_{0}\right]
$$

which is negative because $A_{0}$ corresponds to a direction in $T_{(p, q)} W_{c}$. It follows that the average is positive for $|t|$ small and then (9) holds true. This contradicts the fact that $r$ has a maximum on $V^{k}$ at $(p, q)$. Q.E.D.

COROLlary. Let $M$ be a simply connected, complete Hermitian manifold with nonpositive Riemannian sectional curvature. Then there exist no compact complex analytic subvarieties in $M$.

We observe that when $M$ is Kählerian, this corollary follows from Theorem 1 of [3]. We further note that a (non-Kählerian) Hermitian manifold satisfying the hypothesis of Theorem 3 need not be a Stein manifold, see [4].

\section{REFERENCES}

1. M. Berger, P. Gauduchon et E. Mazet, Le spectre d' une variete Riemannienne, Lecture Notes in Math., vol. 194, Springer-Verlag, New York, 1971. 
2. C. J. Ferraris, On Kähler manifolds with pasitive curoature, J. Differential Geometry 11 (1970), 133-146.

3. R. E. Greene and H. Wu, Curvature and complex analysis, Bull. Amer. Math. Soc. 77 (1971), 1045-1049.

4. P. F. Klembeck, Function theory on complete open Hermitian manifolds, Thesis, Univ. of California, Los Angeles, 1975.

Instituto de Matematica Pura b Aplicada, Rua luiz de Camóes, 68, 20.000 Rio de JANEIRO, R. J., BRASII 